ARTÍCULO DE INVESTIGACIÓN

\title{
Tarifas de biomasa aérea para abedul (Betula pubescens Ehrh.) y roble (Quercus robur L.) en el noroeste de España
}

\author{
Above-ground biomass equations for birch \\ (Betula pubescens Ehrh.) and pedunculate oak \\ (Quercus robur L.) in north western Spain
}

\author{
Esteban Gómez-García ${ }^{1 *}$, Felipe Crecente-Campo ${ }^{1}$ \\ y Ulises Diéguez-Aranda ${ }^{1}$
}

\begin{abstract}
RESUMEN
El objetivo de este trabajo fue el desarrollo de modelos de estimación de biomasa aérea para abedul (Betula pubescens Ehrh.) y roble (Quercus robur L.) en Galicia (noroeste de España). Para ello se emplearon datos obtenidos mediante el muestreo destructivo de 50 abedules y 50 robles, en los que se obtuvo el peso seco de biomasa total y por fracciones: madera del fuste, corteza del fuste, ramas mayores de $2 \mathrm{~cm}$, ramas de $0,5 \mathrm{~cm}$ a $2 \mathrm{~cm}$, ramas menores de $0,5 \mathrm{~cm}$, y hojas. En un primer paso se seleccionaron los modelos que presentaban el mejor ajuste para cada fracción de biomasa arbórea considerada. Posteriormente, y para asegurar la aditividad, se realizó un ajuste simultáneo de las ecuaciones de estimación de biomasa por fracciones junto con la de biomasa total, empleando el procedimiento estadístico denominado NSUR (Nonlinear Seemingly Unrelated Regressions). También fue necesario un ajuste ponderado para corregir la existencia de heterocedasticidad. El número de condición verificó que no existían problemas graves de multicolinealidad. Al final se obtuvo, para cada especie, un sistema de siete ecuaciones de estimación de biomasa aérea para las distintas fracciones y para la biomasa total. Estas ecuaciones explicaron como mínimo $79 \%$ de la variabilidad observada, y en el caso de las ecuaciones de biomasa total $98 \%$ para abedul y $97 \%$ para roble. Se recomienda la utilización de las ecuaciones desarrolladas en este estudio en sustitución de las ecuaciones de biomasa existentes para la región.
\end{abstract}

PALABRAS CLAVE:

Aditividad, ajuste simultáneo, Galicia, heterocedasticidad, multicolinealidad.

\begin{abstract}
The aim of this study was to develop models for above-ground biomass estimation for birch (Betula pubescens Ehrh.) and pedunculate oak (Quercus robur L.) in Galicia (northwest of Spain). Data from a destructive sampling of 50 birch trees and 50 pedunculate oak trees, that provided total dry biomass weight and dry biomass weight by components: wood, bark, branches over $2 \mathrm{~cm}$, branches between $0,5 \mathrm{~cm}$ and $2 \mathrm{~cm}$, branches under $2 \mathrm{~cm}$, and leaves, was used. Different models were fitted for each above-ground component and the best of them was chosen in a first step. All the models were refitted simultaneously by use of Nonlinear Seemingly Unrelated Regressions (NSUR) to ensure the additivity of the biomass equations. Weighted regression was necessary to correct the heterocedasticity of residuals. Not serious problems with multicollineality were observed. Finally, a system of seven equations for the different above-ground biomass components, and for the total biomass, was obtained for
\end{abstract}


each species. These equations explained at least $79 \%$ of the observed variability. For total biomass the equations explained $98 \%$ and $97 \%$ of the observed variability for birch and oak, respectively. The developed biomass equations are recommended in substitution of the existing regional biomass equations.

KEYWORDS:

Addivity, simultaneous fitting, Galicia, heterocedasticity, multicollineality.

\section{INTRODUCCIÓN}

La estimación de la biomasa forestal, así como la acumulación de carbono en la misma, ha ganado importancia en los últimos años como resultado del Protocolo de Kyoto (UNFCCC, 1997), que reconoce que los ecosistemas forestales pueden contribuir a la mitigación del efecto invernadero inducido por las actividades humanas de extracción y emisión de carbono (entre otros elementos) a la atmósfera. Por otra parte, la necesidad de reducir la dependencia de los combustibles fósiles ha traído como consecuencia la consideración de los residuos de las operaciones forestales como una fuente renovable de energía. Las mediciones necesarias para cuantificar la biomasa de un árbol o de un rodal con exactitud son lentas y costosas, por lo que se deben desarrollar ecuaciones que proporcionen estimaciones fiables de la biomasa y que sean aplicables en la gestión forestal práctica. Estas ecuaciones relacionan la biomasa total o por fracciones con variables fáciles de medir en campo.

Los primeros estudios sobre cuantificación de biomasa arbórea surgieron para estimar la producción en peso seco de diferentes especies, destacando entre ellos los relacionados con Larix decidua Mill. y Picea abies (L.) Karst. en Suiza (Burger, 1945; Burger, 1953). En las últimas décadas han aumentado los estudios sobre la estimación de biomasa forestal, alcan- zando cada vez mayor relevancia y abarcando un gran número de especies y estructuras de masa diferentes (p. ej., Pardé, 1980; Cunia, 1986; Zeide, 1987; Waring y Running, 1998; Johansson, 2000; Merino et al., 2003; Cienciala et al., 2005).

A escala europea, Zianis et al. (2005) llevaron a cabo una revisión de las ecuaciones de biomasa desarrolladas para especies forestales de los géneros Abies, Acer, Alnus, Arbutus, Betula, Eucalyptus, Fagus, Fraxinus, Larix, Picea, Pinus, Populus, Pseudotsuga, Quercus y Tilia. Estos autores encontraron 607 ecuaciones de biomasa, una minoría de ellas para el sur del continente. Montero et al. (2005) desarrollaron tarifas de biomasa de árbol individual para algunas de las principales especies forestales españolas (géneros Abies, Alnus, Betula, Castanea, Erica, Ceratonia, Eucalyptus, Fagus, Fraxinus, Ilex, Juniperus, Laurus, Myrica, Olea, Pinus, Populus y Quercus), empleando modelos alométricos y el diámetro normal como variable independiente. En Galicia (nororeste de España, Fig. 1), DiéguezAranda et al. (2009) realizaron una revisión de las ecuaciones de biomasa desarrolladas para la región. Esta revisión incluía tarifas de biomasa de árbol individual para abedul (Betula pubescens Ehrh.) y roble (Quercus robur L.), desarrolladas por Balboa-Murias et al. (2005, 2006, respectivamente); y también para Eucalyptus globulus Labill., E. nitens $\mathrm{H}$. Deane \& Maiden, Pinus radiata D. Don y $P$. pinaster Ait. En el caso del abedul usaron únicamente el diámetro normal como variable independiente, mientras que para roble se usó el diámetro normal y la altura total como variables independientes. El principal problema de las tarifas de biomasa existentes para abedul y roble en Galicia radica en que se ajustaron empleando un reducido número de datos, recogidos únicamente en la provincia de Lugo y en zonas bastante próximas entre si, por lo que su representatividad a nivel 
gallego es limitada. Además, el muestreo de biomasa fue realizado en invierno, por lo que los árboles muestreados no disponían de hojas, y esta fracción la ajustaron con valores estimados.

\section{OBJETIVOS}

El objetivo de este estudio es el desarrollo de nuevas tarifas de biomasa de árbol individual para abedul y roble en Galicia, para lo que se dispuso de una base de datos más completa, que reflejaba mejor la realidad gallega y que contaba con observaciones en la fracción hojas para ambas especies.

\section{METOdologíA}

\section{Datos}

Para la elaboración de ecuaciones de estimación de biomasa aérea se mues- trearon destructivamente un total de 50 abedules y 50 robles, procedentes tanto de regeneración natural como de plantación, repartidos en 11 y 9 localizaciones diferentes, respectivamente, en las provincias de Lugo, Ourense y Pontevedra (Fig. 1). En el invierno de 2002-2003 se cortaron 16 abedules en 2 localizaciones y 31 robles en 4 localizaciones, estos datos son los que emplearon Balboa-Murias et al. $(2005,2006)$ en el desarrollo de sus tarifas de biomasa. En el verano de 2009 se cortaron 34 abedules en 9 localizaciones y 19 robles en 5 localizaciones, con la intención de completar la muestra existente y llegar a los 50 árboles muestreados para cada especie. Se cortaron árboles de diferentes clases sociológicas (dominante, codominante, intermedio y sumergido) que no presentasen malformaciones en el tronco y la copa y sin desarrollos anómalos o desequilibrios motivados por su proximidad a claros o bordes de masa o caminos.

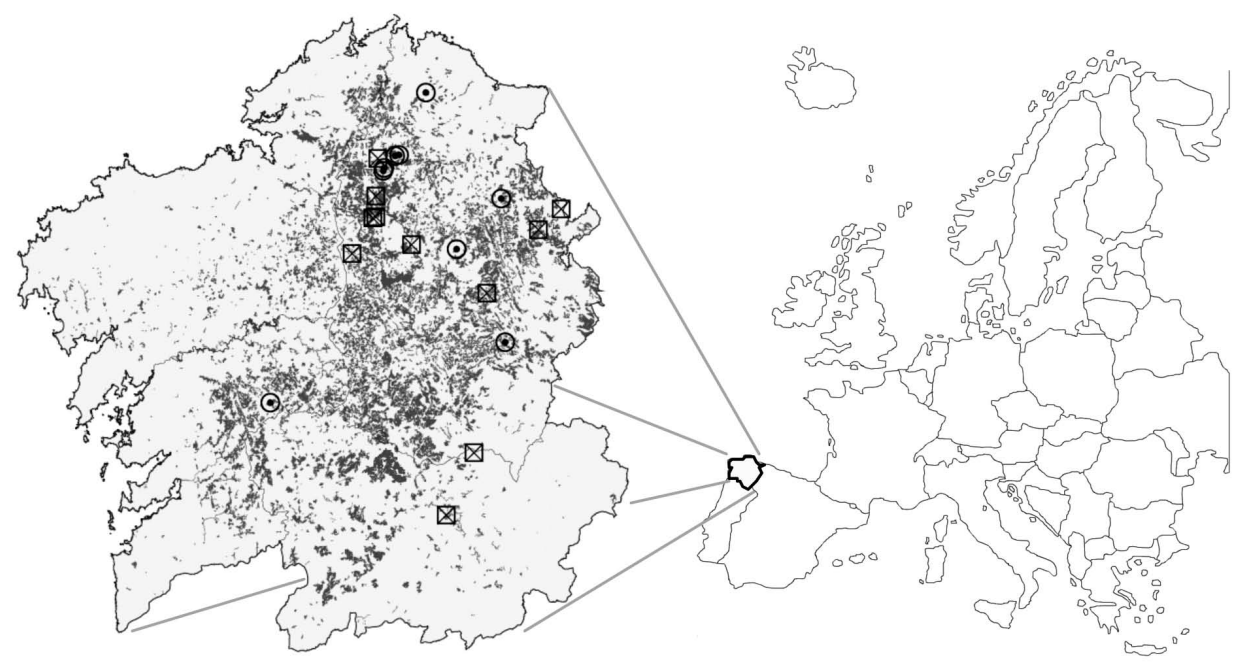

Figura 1. Localización geográfica de Galicia y lugares donde se realizaron los muestreos de biomasa para abedul (cuadrados) y roble (círculos). También se representan los bosques de la región donde dominan estas especies. 
Antes de cortar cada árbol se midió su diámetro normal (a 1,3 m sobre el suelo) en dos direcciones (una siguiendo curvas de nivel y otra en dirección perpendicular a ésta) mediante forcípula (modelo Mantax Blue de 800 mm, Haglöf, Suecia; resolución de $0,1 \mathrm{~cm})$, y su altura total mediante hipsómetro (modelo Vertex IV, Haglöf, Suecia; resolución de 0,1 m). Tras cortar cada árbol se midieron la altura del tocón por la parte ladera arriba del árbol (siendo éste siempre inferior a $10 \mathrm{~cm}$ ) y la longitud del tronco, ambas con cinta métrica. Posteriormente, se dividió la biomasa arbórea aérea en las siguientes fracciones: fuste (hasta 7 $\mathrm{cm}$ en punta delgada), ramas mayores de $7 \mathrm{~cm}$ (diámetro mínimo en punta delgada de $7 \mathrm{~cm}$ ), ramas gruesas (diámetro máximo en punta gruesa de $7 \mathrm{~cm}$ y mínimo en punta delgada de $2 \mathrm{~cm}$ ) y ramas menores de $2 \mathrm{~cm}$ (incluyendo hojas). Estas fracciones se pesaron en campo con una báscula GRAM modelo K3-300L (plataforma de 600 $\mathrm{mm}$ x $600 \mathrm{~mm}, 300 \mathrm{~kg}$ de capacidad y $50 \mathrm{~g}$ de resolución).

A continuación se recogieron muestras de cada fracción para llevar a laboratorio. En el fuste se cortaron tres discos transversales de $4 \mathrm{~cm}$ de grosor (el fuste se consideró dividido en tres trozas de igual longitud y se seleccionó una rodaja en el centro de cada una de ellas). Se recogió una muestra aleatoria de ramas mayores de $7 \mathrm{~cm}$ y una muestra aleatoria de ramas gruesas (aproximadamente 1,5 $\mathrm{kg}$ en cada una de estas fracciones). En las ramas menores de $2 \mathrm{~cm}$ se tomó al azar una muestra de $6 \mathrm{~kg}$ ( $2 \mathrm{~kg}$ en la parte baja de la copa, $2 \mathrm{~kg}$ en la parte media y 2 $\mathrm{kg}$ en la parte alta). Para evitar en lo posible la pérdida de humedad, estas muestras fueron transportadas en sacos de plástico cerrados.

Las muestras se procesaron en laboratorio el mismo día de la corta de cada pie. La muestra de ramas menores de 2 $\mathrm{cm}$ se dividió en ramas finas (diámetro máximo en punta gruesa de $2 \mathrm{~cm}$ y mínimo en punta delgada de $0,5 \mathrm{~cm}$ ), ramillos (diámetro máximo en punta gruesa de 0,5 $\mathrm{cm}$ ) y hojas (si las hubiese). Las rodajas del fuste se dividieron en madera y corteza. La proporción, en peso verde, de estas nuevas fracciones se aplicó al árbol completo, por lo que se consideraron siete fracciones: madera (del fuste), corteza (del fuste), ramas mayores de $7 \mathrm{~cm}$, ramas gruesas $(2 \mathrm{~cm}-7 \mathrm{~cm})$, ramas finas $(0,5 \mathrm{~cm}-2 \mathrm{~cm})$, ramillos $(<0,5 \mathrm{~cm})$ y hojas.

El contenido en humedad de las siete fracciones consideradas se determinó en laboratorio a partir de las muestras recogidas en campo. Se empleó la totalidad de la cantidad disponible en cada fracción, salvo en las ramas finas, ramillos y hojas, considerando suficiente secar aproximadamente $1,5 \mathrm{~kg}, 1,0 \mathrm{~kg}$ y $0,5 \mathrm{~kg}$ respectivamente. Las muestras fueron pesadas en verde con balanza de precisión GRAM modelo SBZ1500 (1500 g de capacidad y 0,01 g de resolución). Posteriormente se secaron en una estufa LABOLAN-INDELAB modelo ECOLAN IDL.FI720 (720 I de capacidad y dimensiones interiores de $120 \mathrm{~cm} \times 100 \mathrm{~cm} \times 60$ $\mathrm{cm}$ ) a $65^{\circ} \mathrm{C}$ hasta peso constante (cuando dos mediciones realizadas con una separación de 12 horas difieren menos de 0,5\% en peso). El contenido en humedad se calculó en función de los pesos verde y seco. A partir del contenido en humedad se obtuvo el peso seco total de cada fracción, que fue el utilizado para la elaboración de las ecuaciones de biomasa.

El muestreo de biomasa del año 2002-2003 fue realizado en invierno, por lo que los árboles muestreados no disponían de hojas. Por tal motivo, el ajuste de la ecuación de estimación de biomasa para esta fracción se realizó únicamente a partir de los datos de los 34 abedules y de los 19 robles muestreados en el verano de 2009. Con respecto a las fracciones de ramas finas y ramillos de abedul, los datos correspondientes al primer muestreo mos- 

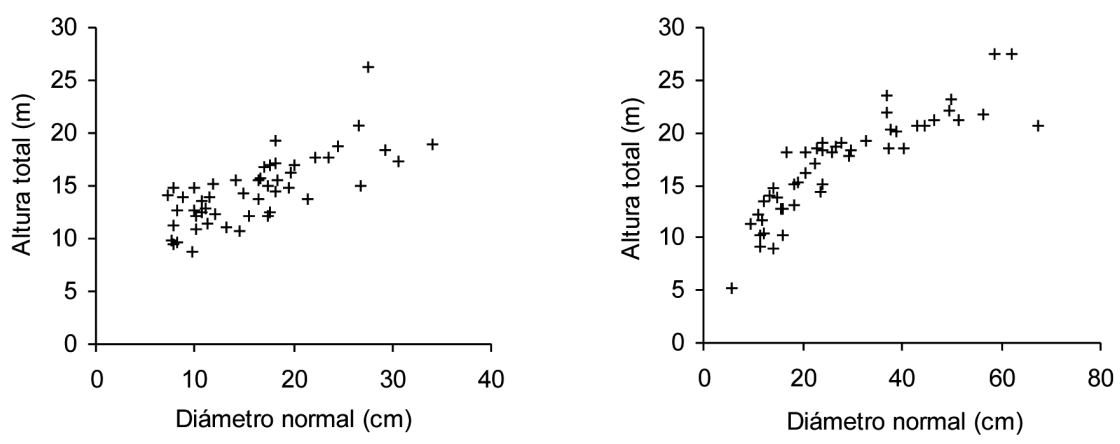

Figura 2: Distribución altura-diámetro de los árboles de B. pubescens (izquierda) y $Q$. robur (derecha) empleados en los ajustes.

traron una tendencia anómala, siendo imposible investigar la causa, aunque podría ser motivado por deficiencias en el proceso de secado; por este motivo, el ajuste de las ecuaciones de estimación de biomasa de estas dos fracciones se realizó a partir de los datos de los 34 abedules muestreados en 2009. En la figura 2 se representa, para cada especie, la distribución altura-diámetro de los árboles empleados en los ajustes. En la tabla 1 se muestran, para cada especie, los estadísticos descriptivos de las variables diámetro normal, altura total y peso seco de biomasa aérea total y por fracciones.

\section{Ajuste de las ecuaciones de biomasa}

La metodología empleada en el ajuste de las ecuaciones de biomasa fue similar a la que utilizaron Balboa-Murias et al. (2006) para $Q$. robur en Galicia. Una vez determinada la biomasa seca de las diferentes fracciones de los árboles muestreados, se desarrollaron ecuaciones que relacionen dichos pesos con una o más variables independientes. Estas variables independientes deben ser: (i) poco numerosas y fáciles de medir para que el modelo tenga una amplia gama de aplicaciones y su empleo sea sencillo, (ii) variables fuerte- mente correlacionadas con la biomasa, y (iii) variables poco correlacionadas entre sí para evitar problemas de multicolinealidad.

El diámetro normal es la variable más utilizada en las ecuaciones de estimación de biomasa arbórea $(w=f(d))$, aunque añadiendo la altura del árbol como segunda variable explicativa $(w=f(d, h))$ suelen mejorar significativamente los ajustes (Wang, 2006). Como variables independientes adicionales al diámetro y a la altura se pueden emplear otras variables de árbol, como la longitud de la copa viva (diferencia entre la altura total y la altura a la que se localiza la primera rama viva) o el diámetro en la base de la copa, que han resultado especialmente útiles para mejorar las estimaciones de biomasa de las fracciones de copa (p. ej., Clark, 1982; Satoo y Madgwick, 1982). También se pueden emplear variables de rodal como la edad, el área basimétrica, la altura dominante o la altura media (p. ej., Satoo y Madgwick, 1982; Bond-Lamberty et al., 2002; Porté et al., 2002). Estas opciones presentan como inconveniente que para emplear las ecuaciones de biomasa es necesario un mayor esfuerzo de inventario $\mathrm{y}$, además, las ecuaciones desarrolladas presentan un intervalo de aplicación más reducido. 


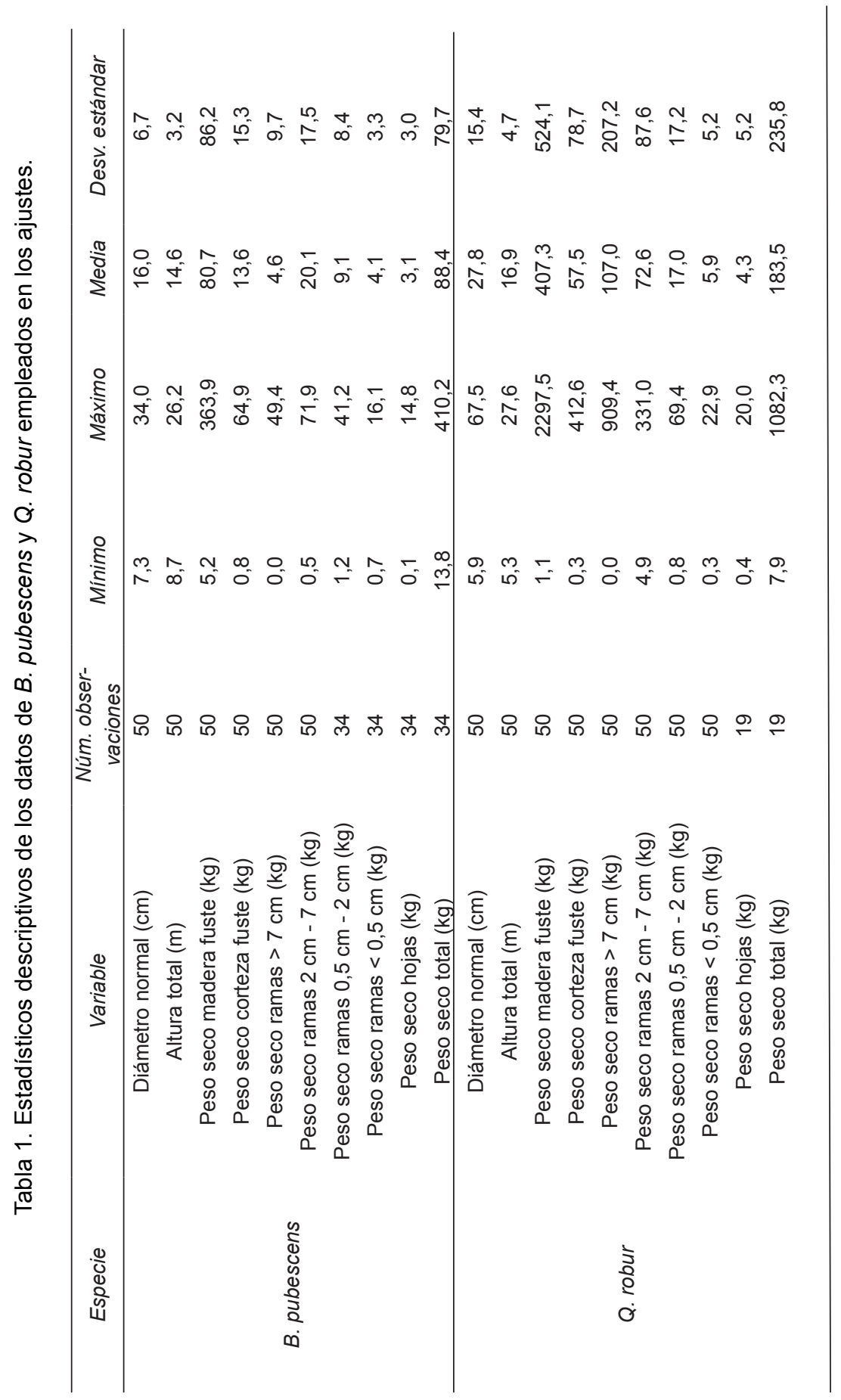


La selección del modelo a emplear para la estimación de la biomasa de cada fracción ha de buscar un equilibrio entre la precisión, la sencillez y la aplicación práctica (Wang, 2006). Aunque existe una gran variedad de modelos válidos para estimar la biomasa, todos ellos derivan de alguna de las tres formas matemáticas siguientes (Pardé, 1980; Snowdon, 1985; Parresol, 1999):

Lineal (error aditivo):

$$
Y=\beta_{0}+\beta_{1} X_{1}+\ldots+\beta_{j} X_{j}+\varepsilon
$$

No lineal (error aditivo):

$$
Y=\beta_{0} X_{1}^{\beta_{1}} X_{2}^{\beta_{2}} \ldots X_{j}^{\beta_{j}}+\varepsilon
$$

No lineal (error multiplicativo):

$$
Y=\beta_{0} X_{1}^{\beta_{1}} X_{2}^{\beta_{2}} \ldots X_{j}^{\beta_{j}} \varepsilon
$$

donde:

$Y$ : biomasa total o de una fracción determinada

$X_{j}$ : variables explicativas

$\beta_{i}$ : parámetros del modelo

$\varepsilon$ : término del error

El modelo (1) se ajusta por regresión lineal, el modelo (2) requiere emplear una técnica iterativa de regresión no lineal y el modelo (3) se ajusta por regresión lineal realizando una transformación logarítmica de ambos términos de la igualdad.

En este trabajo, en un primer paso, se seleccionó para cada especie y para cada fracción de biomasa el modelo que presentaba una mejor capacidad de ajuste. Los modelos evaluados emplean como variables independientes únicamente variables de árbol individual (diámetro normal y, en ocasiones, altura total). Se ajustaron modelos lineales y alométricos (modelos (1) y (2)). Los modelos lineales se ajustaron por mínimos cuadrados empleando el procedi- miento REG del programa SAS/STAT ${ }^{\circledR}$ (SAS Institute Inc., 2009) y los modelos no lineales se ajustaron por mínimos cuadrados empleando el procedimiento NLIN del mismo programa, usando el método iterativo de Gauss-Newton. En un segundo paso las ecuaciones de estimación de biomasa por fracciones y de biomasa total se ajustaron simultáneamente para garantizar la aditividad.

Inicialmente se ajustaron modelos para cada fracción muestreada, obteniéndose resultados pocos satisfactorios para la fracción de ramas mayores de $7 \mathrm{~cm}$ en cualquiera de las dos especies, presentando además el ajuste simultáneo problemas de convergencia. Por ello, y teniendo en cuenta que no todos los pies disponían de datos de esta fracción, las ramas mayores de $7 \mathrm{~cm}$ se integraron en la fracción de ramas gruesas. Así las ramas gruesas pasaron a ser ramas con un diámetro mayor de $2 \mathrm{~cm}$.

\section{Heterocedasticidad y multicolinealidad}

Es frecuente que en el desarrollo de tarifas de biomasa las estimaciones presenten problemas de heterocedasticidad, es decir que la varianza de los errores no sea constante (Parresol, 2001); y este fenómeno invalida los contrastes de significación de los parámetros. Para analizar la presencia de heterocedasticidad se analizaron los gráficos de residuos estudentizados (Mendoza et al., 2002) frente a valores estimados y se calculó el contraste de White (1980), que es independiente de la forma funcional del modelo y del tipo de distribución de datos. Para corregir la presencia de heterocedasticidad se empleó regresión ponderada, como en otros trabajos de estimación de biomasa arbórea (p. ej., Schlaegel, 1982; Cunia, 1986; Parresol, 1999). Así, en la fase de ajuste, es necesario ponderar cada observación con un peso igual a la 
inversa de la varianza de su error $\left(\sigma_{1}^{2}\right)$. Dicha varianza es desconocida, pero con frecuencia al examinar los gráficos de residuos frente a las variables independientes se observa que esta varianza se incrementa cuando se incrementa el valor de alguna o todas las variables independientes $(X)$; por tanto se puede estimar utilizando una función potencial del tipo $\sigma_{i}^{2}=X_{i}^{k}$ (Neter et al., 1996). El valor del exponente $k$ se puede determinar empleando la metodología propuesta por Park (1966), que consiste en utilizar los errores del modelo ajustado sin pesos $\hat{e}_{i}$ como variable dependiente en el modelo potencial de varianza del error, es decir:

$$
\begin{aligned}
& \hat{e}_{i}^{2}=\gamma X_{i}^{k} \quad \text { obien } \\
& \qquad \ln \hat{e}_{i}^{2}=\ln \gamma+k \ln X_{i}
\end{aligned}
$$

En función de las variables independientes que interviniesen en cada modelo se probaron distintos factores de ponderación (p. ej., $d^{-k},\left(d^{2} h\right)^{-k}$, etc.).

Otro problema frecuente en el ajuste de ecuaciones de biomasa es la presencia de multicolinealidad (fuerte correlación entre las variables independientes). Sus efectos son similares a los indicados para la heterocedasticidad, invalidando también los contrastes de significación. Cuando los problemas de multicolinealidad son graves el modelo no es robusto y la estimación de parámetros depende en exceso del conjunto particular de datos utilizado en el ajuste (Myers, 1990). Se puede evaluar la presencia de multicolinealidad en cada uno de los modelos ajustados calculando el número de condición (NC):

$$
\mathrm{NC}=\left(\frac{\text { máximo autovalor matriz } R}{\text { mínimo autovalor matriz } R}\right)^{0,5}
$$

donde $R$ es la matriz de correlaciones entre las variables independientes del modelo.
Cuando las variables definen un sistema ortogonal todos los autovalores son iguales a 1 , mientras que cuando existe un alto grado de correlación entre variables algún autovalor es próximo a cero. De acuerdo con Belsley (1991, pp. 139$141)$, si $N C<10$ no hay problemas de multicolinealidad, si $30 \leq \mathrm{NC} \leq 100$ existen problemas de multicolinealidad y si $1000<$ $\mathrm{NC}<3000$ los problemas son graves.

\section{Aditividad y ajuste simultáneo}

Una de las propiedades más importantes que deben cumplir las ecuaciones de estimación de biomasa de las distintas fracciones en que se divide un árbol es que la suma de sus estimaciones sea igual a la estimación obtenida por la ecuación de estimación de biomasa total, propiedad que se denomina aditividad. Aunque existen varios procedimientos que se pueden utilizar para forzar esta propiedad en un sistema de ecuaciones (Parresol, 1999; Balboa-Murias et al., 2004), el ajuste simultáneo es el más flexible y general; puesto que permite usar diferentes modelos matemáticos para cada fracción, con distintas variables independientes y funciones de ponderación. Se basa en el ajuste de un sistema de ecuaciones, aparentemente no relacionadas, formado por las $k$ fracciones arbóreas consideradas junto con la de biomasa total.

$$
\begin{gathered}
\hat{w}_{1}=f_{1}\left(x_{1}\right) \\
\hat{w}_{2}=f_{2}\left(x_{2}\right) \\
\cdot \\
\cdot \\
\hat{w}_{k}=f_{k}\left(x_{k}\right) \\
\hat{w}_{\text {total }}=f_{\text {total }}\left(x_{1}, x_{2}, \ldots, x_{k}\right)
\end{gathered}
$$


donde:

$\hat{w}_{k}$ : biomasa estimada

$x_{k}$ : conjunto de variables explicativas

En este caso, las variables independientes del modelo de biomasa total son todas las variables independientes que aparecen en los restantes modelos, y la aditividad está garantizada imponiendo restricciones a los parámetros del modelo de biomasa total. Este sistema, sin relaciones analíticas entre ecuaciones, se suele resolver empleando regresión SUR (Seemingly Unrelated Regression) también conocida por mínimos cuadrados generalizados conjuntos o regresión Zellner (Zellner, 1962). Para sistemas de ecuaciones no lineales este método se denomina NSUR y es igual de válido que para ecuaciones lineales. Otra ventaja de esta metodología radica en corregir el problema de dependencia de errores entre las estimaciones de cada fracción de biomasa, considerando que no es realista asumir que los errores de cada ecuación no estén correlacionados (Borders, 1989; Parresol, 1999, 2001).

Los parámetros de todas las ecuaciones fueron obtenidos de forma simultánea utilizando la metodología NSUR, para lo que se utilizó el procedimiento MODEL del programa SAS/ETS ${ }^{\circledR}$ (SAS Institute Inc., 2008). Se realizó un ajuste simultáneo inicial y a partir de los residuos obtenidos de éste se calcularon los pesos a utilizar en la ponderación del ajuste simultáneo definitivo, con la finalidad de corregir la heterocedasticidad y garantizar la aditividad.

\section{Ajuste en dos pasos}

Como ya se ha mencionado, en el caso del abedul algunos árboles no disponían de datos de las fracciones de ramas finas, ramillos y hojas (lo que en conjunto se denominó "copa fina"), debido a que no existían o a que fueron descartados por presentar anomalías. Sin embargo, el resto de fracciones presentaban tendencias lógicas, por lo que se decidió utilizarlas en los ajustes. Para solucionar este inconveniente, en un paso previo se realizó un ajuste simultáneo de las ecuaciones de estas tres fracciones, más una cuarta que las engloba (biomasa de copa fina), a partir de los datos de los 34 árboles muestreados en el verano de 2009. Para obtener las estimaciones de los parámetros del resto de ecuaciones se realizó un ajuste simultáneo del sistema constituido por las ecuaciones de biomasa de fuste, de corteza, de ramas gruesas y de biomasa total (incluyendo en ésta la biomasa estimada de copa fina).

En el caso del roble, al no existir la fracción de hojas para algunos árboles, se empleó un procedimiento similar. En primer lugar se ajustó esta fracción de forma independiente, a partir de los 19 árboles muestreados en el verano de 2009. Posteriormente se ajustaron el resto de ecuaciones de forma simultánea (fuste, corteza, ramas gruesas, ramas finas, ramillos y biomasa total -incluyendo en ésta la biomasa estimada de las hojas).

\section{Criterios de selección de modelos}

La selección del mejor modelo para cada fracción se ha basado en el análisis de la capacidad de ajuste, tanto por métodos analíticos como gráficos. Así, a partir de los residuos, se calcularon dos estadísticos utilizados con frecuencia en modelización forestal: el coeficiente de determinación $\left(R^{2}\right)$ y la raíz del error medio cuadrático (REMC); cuyas expresiones son las siguientes:

$$
R^{2}=1-\frac{\sum_{i=1}^{i=n}\left(Y_{i}-\hat{Y}\right)^{2}}{\sum_{i=1}^{i=n}\left(Y_{i}-\bar{Y}\right)^{2}}
$$




$$
\mathrm{REMC}=\sqrt{\frac{\sum_{i=1}^{i=n}\left(Y_{i}-\hat{Y}_{i}\right)^{2}}{n-p}}
$$

donde:

$Y_{i}$ : valor observado de la variable dependiente

$\hat{Y}_{i}$ : valor estimado de la variable dependiente

$\bar{Y}$ : valor promedio de la variable dependiente

$n$ : número total de observaciones utilizado para ajustar el modelo

$p$ : número de parámetros a estimar

Aunque existen opiniones fundamentadas que plantean dudas en relación al empleo del $R^{2}$ en la selección de modelos, este estadístico da una idea bastante intuitiva de la variabilidad que explican los modelos, y la utilidad general de alguna medida global de la adecuación del modelo parece anular alguna de estas limitaciones (Ryan, 1997, p. 424); no obstante no debe utilizarse como único criterio para elegir el mejor modelo (Myers, 1990, p. 166). El estadístico REMC resulta útil porque está expresado en las mismas unidades que la variable dependiente, por lo que da una idea del error medio que se comete con el modelo; además penaliza los modelos con mayor número de parámetros, de acuerdo con el principio general de simplicidad científica (Peña, 2002, p. 570).

Además del cálculo de estadísticos de ajuste, una herramienta muy útil para determinar si los ajustes se adecuan a los datos son los análisis gráficos (Huang, 2002). Se realizó una representación gráfica de los valores reales de las variables frente a los predichos, a los que se superpuso la recta ideal $1: 1$, con el fin de inspeccionar de manera visual si las predicciones eran acordes con los valores reales. Por último, se analizaron también los gráficos de residuos estudentizados frente a valores predichos con la finalidad de detectar valores atípicos, tendencias extrañas de los datos o la existencia de problemas de heterocedasticidad.

\section{RESULTADOS Y DISCUSIÓN}

Los modelos que mostraron los mejores resultados para la estimación de la biomasa aérea total y por fracciones para abedul y roble se muestran en la tabla 2 . En la tabla 3 se muestran las estimaciones de los parámetros obtenidas mediante el ajuste simultáneo ponderado, así como sus correspondientes errores estándar aproximados y contrastes de significación. Todos los parámetros resultaron significativos al nivel de $5 \%$, salvo el parámetro $\beta_{61}$ en roble, aunque de forma visual se comprobó la idoneidad del modelo elegido y la necesidad del parámetro en cuestión, por lo que éste se mantuvo en el modelo. También se muestran los pesos utilizados en la ponderación, los estadísticos de bondad de ajuste y el valor del contraste de White, que indica que en todos los casos el ajuste se realizó en condiciones de homogeneidad de la varianza de los errores. El análisis gráfico de los residuos estudentizados (no mostrado) corroboró estos resultados.

En general, las ecuaciones mostraron un ajuste satisfactorio, explicando como mínimo $79 \%$ de la variabilidad observada. En el caso del abedul, cinco de las siete ecuaciones desarrolladas explicaron más de $90 \%$ de la variabilidad de los datos observados. Con respecto al roble, sólo dos ecuaciones superaron este nivel. Cabe destacar, en ambos casos, el buen ajuste que presentan las ecuaciones de biomasa de madera de fuste y de biomasa total, con valores del $R^{2}$ en torno a $95 \%-98 \%$ para ambas especies. 
Tabla 2. Modelos seleccionados para la estimación de la biomasa aérea total y por fracciones para B. pubescens y Q. robur en Galicia.

\begin{tabular}{|c|c|c|}
\hline Especie & Fracción & Modelo \\
\hline \multirow{8}{*}{ B. pubescens } & Madera fuste & $w_{m}=\beta_{10}+\beta_{11} d^{2} h$ \\
\hline & Corteza fuste & $w_{c}=\beta_{2 \lambda} d^{\beta_{23}} h^{\beta_{2}}$ \\
\hline & Ramas gruesas & $w_{r>2}=\beta_{31} d^{\beta_{32}}$ \\
\hline & Ramas finas & $w_{r 05-2}=\beta_{41} d^{\beta_{42}}$ \\
\hline & Ramillos & $w_{r<05}=\beta_{51} d^{2}$ \\
\hline & Hojas & $w_{h}=\beta_{61} d^{2} h$ \\
\hline & Copa fina & $w_{\text {copa fina }}=\beta_{41} d^{\beta_{42}}+\beta_{51} d^{2}+\beta_{61} d^{2} h$ \\
\hline & Total & $w_{\text {total }}=\beta_{10}+\beta_{11} d^{2} h+\beta_{21} d^{\beta_{22}} h^{\beta_{33}}+\beta_{31} d^{\beta_{22}}+w_{\text {copa fina }}$ \\
\hline \multirow{7}{*}{ Q. robur } & Madera fuste & $w_{m}=\beta_{10}+\beta_{11} d^{2} h$ \\
\hline & Corteza fuste & $w_{c}=\beta_{21} d^{2} h$ \\
\hline & Ramas gruesas & $w_{r>2}=\beta_{31} d^{2} h$ \\
\hline & Ramas finas & $w_{r 05-2}=\beta_{41} d^{\beta_{42}}$ \\
\hline & Ramillos & $w_{r<05}=\beta_{50}+\beta_{51} d$ \\
\hline & Hojas & $w_{h}=\beta_{61} d^{\beta_{62}}$ \\
\hline & Total & $w_{\text {fotal }}=\beta_{10}+\beta_{50}+\left(\beta_{11}+\beta_{21}+\beta_{31}\right) d^{2} h+\beta_{41} d^{\beta_{42}}+\beta_{51} d+w_{h}$ \\
\hline $\begin{array}{ll}w_{k}= & \text { peso seco } \\
\beta_{i j}= & \text { parámetro } \\
d= & \text { diámetro } \\
h= & \text { altura tota }\end{array}$ & $\begin{array}{l}\text { as distintas fracci } \\
\text { la fracción arbór } \\
\text { al del árbol }(\mathrm{cm}) \\
\text { árbol }(\mathrm{m})\end{array}$ & $\begin{array}{l}\text { nes o total de biomasa arbórea }(\mathrm{kg}) \\
\text { a } i\end{array}$ \\
\hline
\end{tabular}




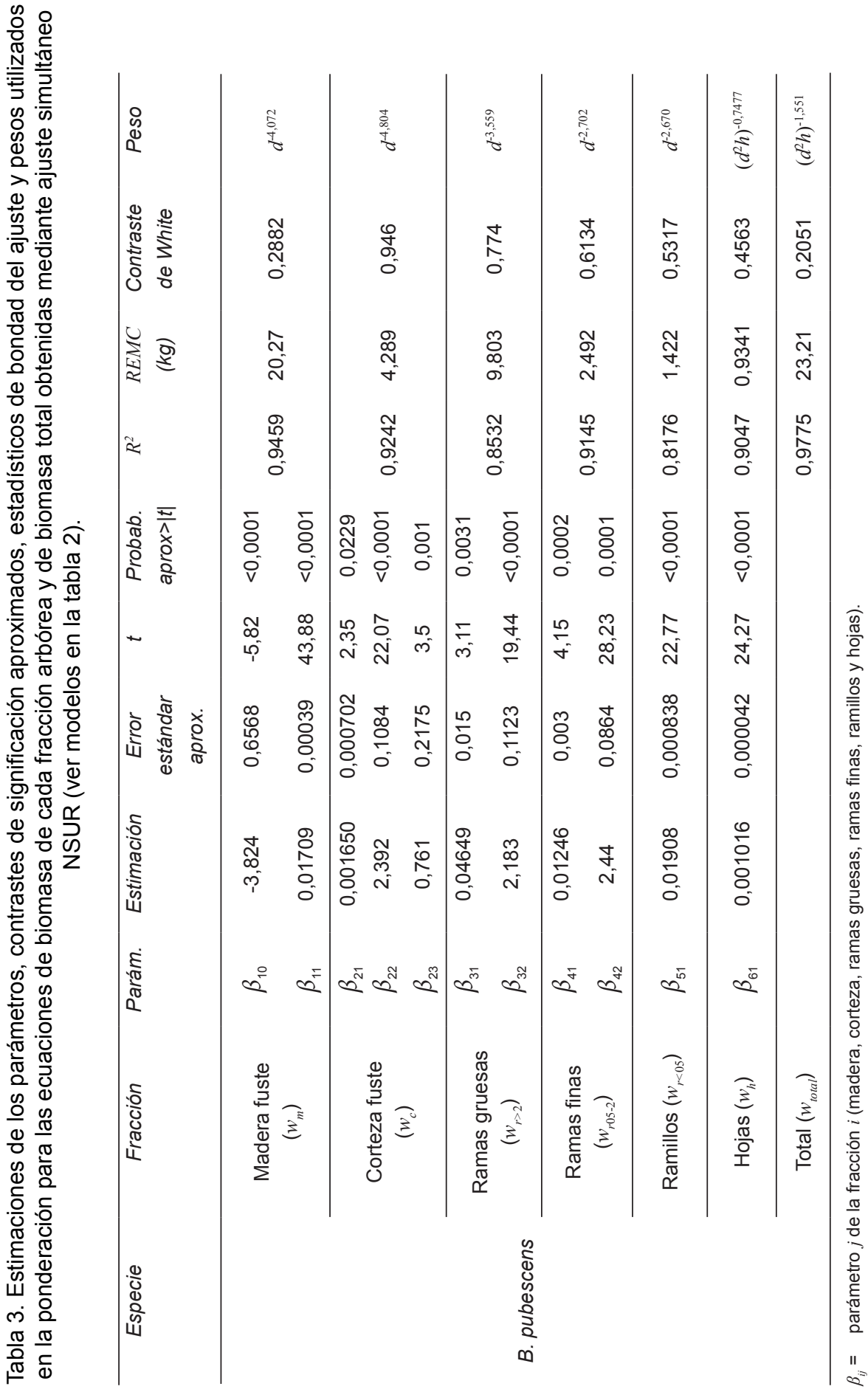


\begin{tabular}{l|}
$m$ \\
$\frac{\pi}{0}$ \\
$\frac{\pi}{\pi}$ \\
$\frac{\pi}{0}$ \\
0 \\
$\frac{3}{0}$ \\
0 \\
0 \\
0
\end{tabular}

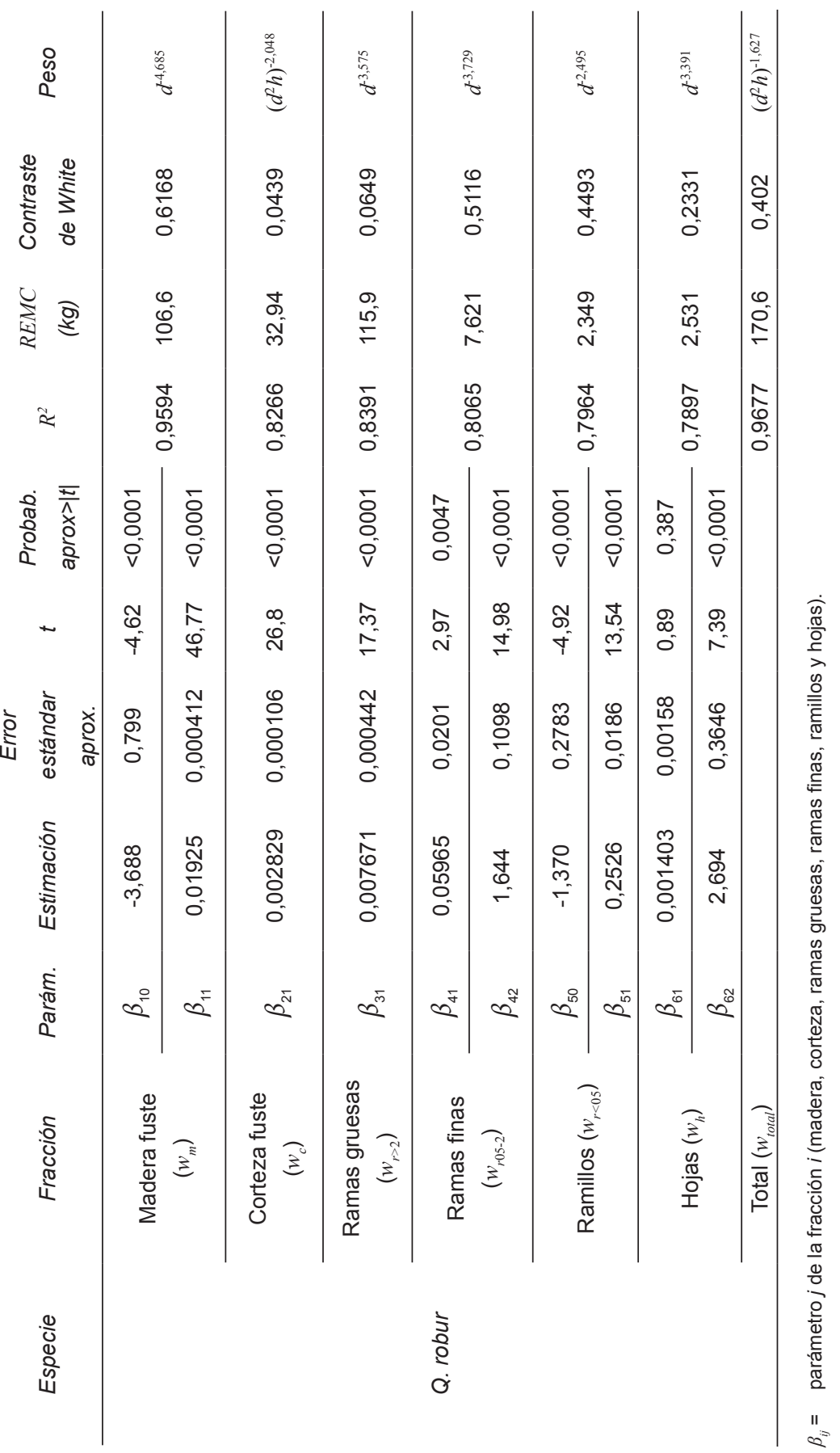


Sin embargo, para el caso del roble, la REMC fue superior siempre, aunque los valores no son directamente comparables, puesto que los valores medios de biomasa fueron mayores en roble que en abedul (Tabla 1). Considerando las mismas fracciones, los estadísticos de ajuste de las ecuaciones desarrolladas en este estudio fueron superiores (mayores valores de $R^{2} y$ menores valores de REMC) a los obtenidos en estudios previos para las mismas especies en la región (Balboa-Murias et al., 2005; Balboa-Murias et al., 2006), con la excepción de la fracción hojas en roble. En esta fracción el valor de $R^{2}$ fue mayor en el estudio de Balboa-Murias et al. (2006) $-R^{2}$ $=0,832$ frente al valor de 0,789 obtenido en este estudio -, pero a su vez también el valor de REMC fue mayor (11,2 kg frente al valor de 2,53 kg obtenido en este estudio). Además, también se debe considerar que en los estudios previos la fracción hojas se ajustó con valores estimados.

En relación con la biomasa de las fracciones de copa (ramas gruesas, ramas finas, ramillos y hojas), éstas presentaron un peor ajuste que la biomasa del fuste. El mismo resultado ha sido constatado en estudios similares, especialmente en el caso de frondosas (Satoo y Madgwick, 1982), lo que posiblemente sea debido a la variabilidad de la estructura de la copa, el número de ramas y la variación de la densidad de la madera a lo largo de las ramas (Pardé, 1980). La inclusión de otra variable, como la longitud de copa viva, podría mejorar el ajuste de estas fracciones, aunque a expensas de un mayor esfuerzo de muestreo para poder aplicar los modelos.

El ajuste simultáneo mediante NSUR proporcionó estimaciones de los parámetros y estadísticos de bondad del ajuste muy similares a los obtenidos mediante el ajuste individual; sin embargo, los errores estándar aproximados disminuyeron sensiblemente cuando se empleó NSUR (resultado directo de considerar la correlación entre los errores de las ecuaciones de biomasa), por lo que se logró una mayor eficiencia en la estimación de parámetros con esta metodología. En otros estudios se obtuvieron resultados similares, verificándose también que el método de estimación NSUR mejora la precisión en las estimaciones (Parresol, 1999; Parresol, 2001; Carvalho y Parresol, 2003; Návar et al., 2004). El número de condición en el ajuste simultáneo ponderado fue de 41,2 para abedul y 16,7 para roble, con lo que se puede concluir que no se presentan problemas graves de multicolinealidad entre las variables independientes.

En las figuras 3 y 4 se muestran los gráficos de valores observados frente a predichos para las distintas fracciones de biomasa consideradas y para la biomasa total. Las nubes de puntos se sitúan cerca de la diagonal $1: 1$, indicando que los modelos proporcionan estimaciones con bajo sesgo. En el caso del roble, y para la biomasa de ramas gruesas, aparece una ligera tendencia a la subestimación para los mayores valores. Esta ecuación es una de las que presenta mayor valor de REMC $(115,9 \mathrm{~kg})$.

La proporción media de biomasa de las fracciones madera del fuste, corteza del fuste, ramas (ramas gruesas, ramas finas y ramillos) y hojas con respecto a la biomasa aérea total fue, respectivamente, de $53,3 \% ; 8,9 \% ; 34,2 \%$ y $3,6 \%$ en abedul y de $57,1 \% ; 9,1 \% ; 31,2 \%$ y $2,6 \%$ en roble. Resultados similares fueron obtenidos en estudios previos en Galicia (BalboaMurias et al., 2005; Balboa-Murias et al., 2006). Ambas especies presentan porcentajes similares de las mismas fracciones, con una proporción de las fracciones que forman la copa entre $34 \%$ y $38 \%$, mayor que otras especies para las que se han desarrollado ecuaciones de estimación de biomasa en la región ( $E$. globulus, $E$. nitens, $P$. radiata y $P$. pinaster) con una proporción de biomasa de copa entre 10\% y 19\% (Diéguez Aranda et al., 2009). 

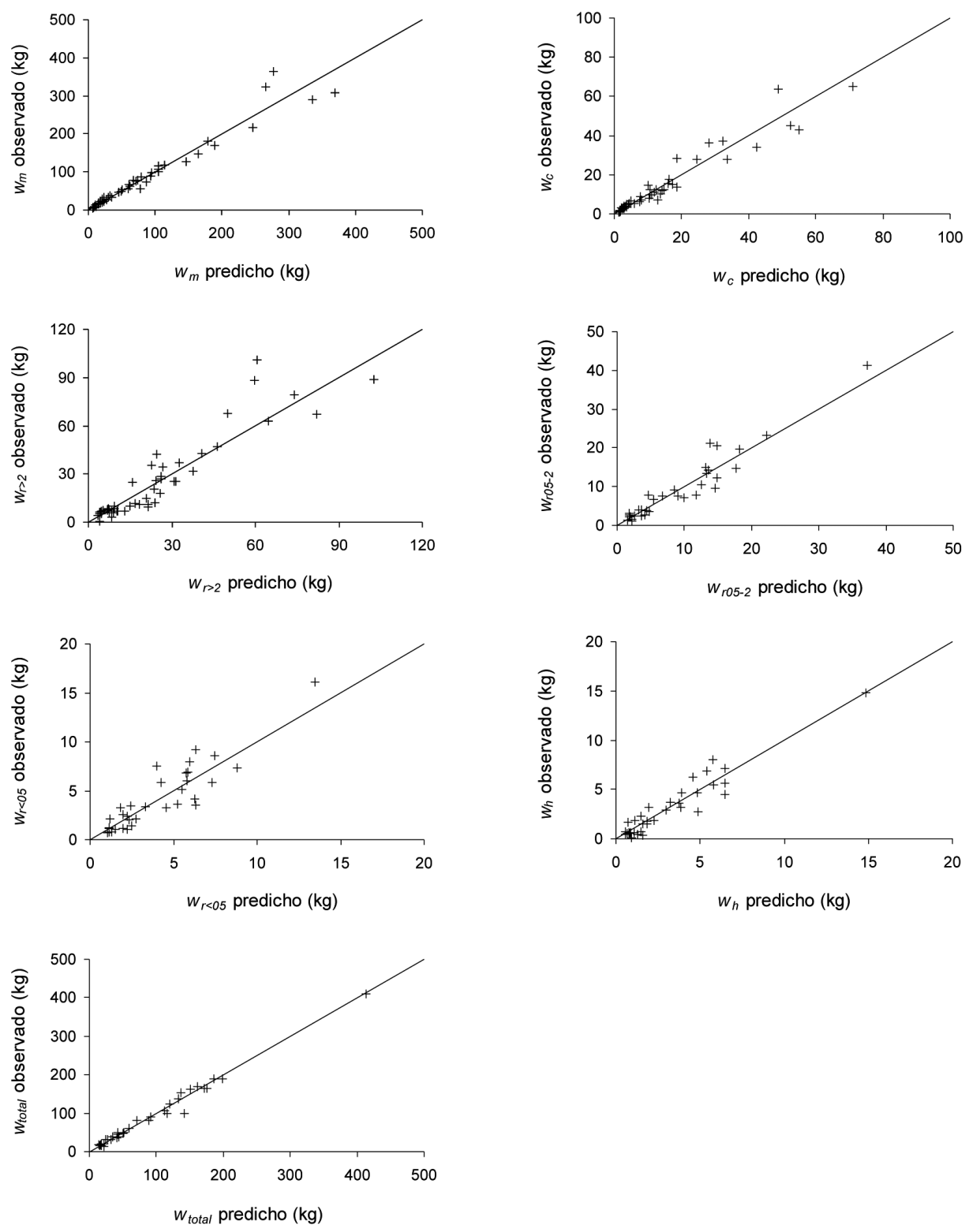

Figura 3. Valores observados frente a predichos para las distintas fracciones de biomasa y para la biomasa total de B. pubescens (variables definidas en Tabla 2).

Los gráficos de biomasa de ramas finas, ramillos, hojas y biomasa total sólo incluyen datos de los 34 árboles muestreados en verano de 2009. 

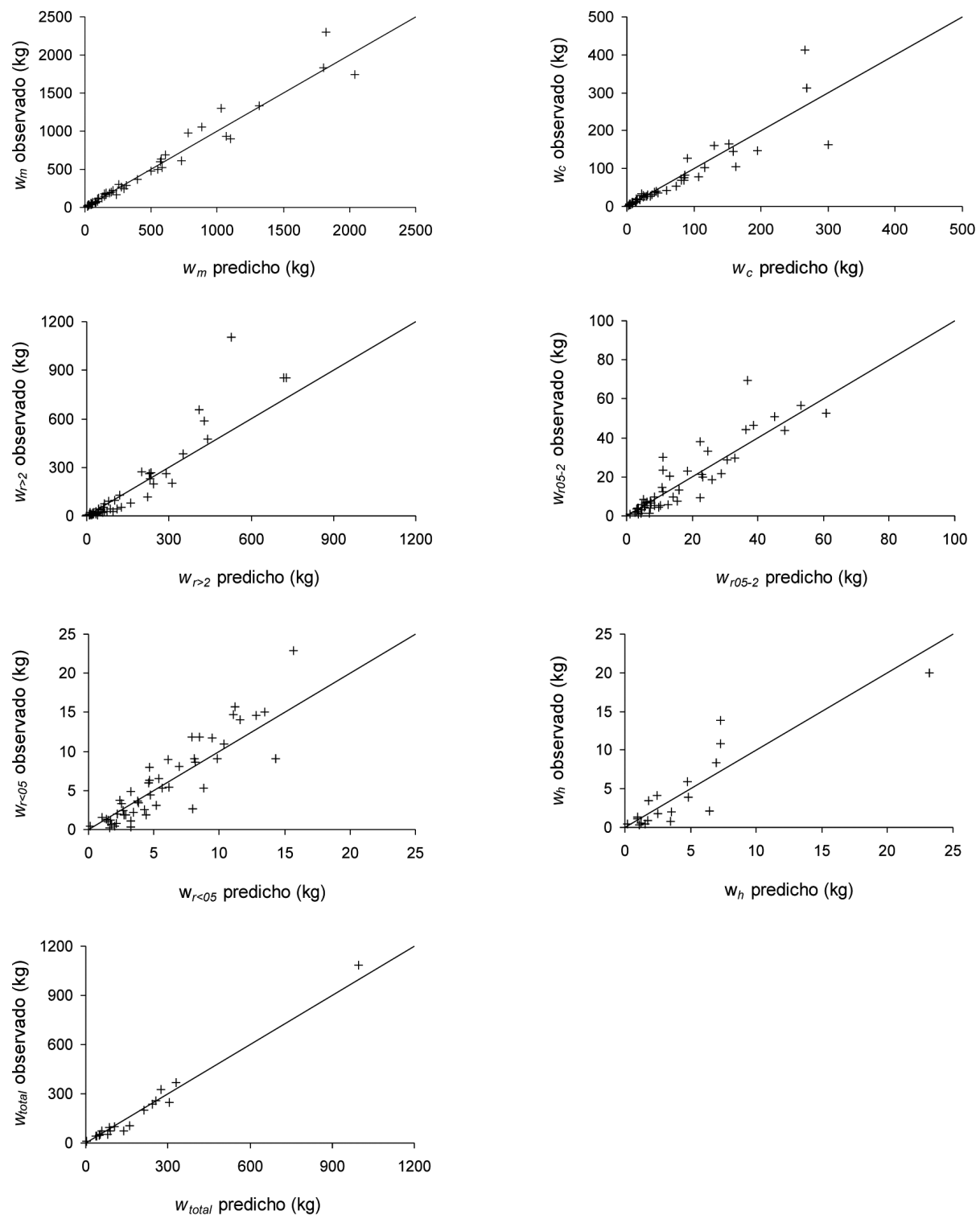

Figura 4. Valores observados frente a predichos para las distintas fracciones de biomasa y para la biomasa total de $Q$. robur (variables definidas en Tabla 2).

Los gráficos de biomasa de hojas y biomasa total sólo incluyen datos de los 19 árboles muestreados en verano de 2009. 
En abedul las fracciones se agrupan en una proporción de biomasa de fuste de $62,2 \%$ y de biomasa de copa del $37,8 \%$. Estos resultados son acordes con los obtenidos en otros estudios para especies del mismo género en España (Gracia et al., 2002) y Norteamérica (Jokela et al., 1981; Schmitt y Grigal, 1981; Maliondo et al., 1990; Jian et al., 1998), pero difieren de los obtenidos por Marklund (1988) para Suecia, en donde no se consideraba la fracción hojas y las proporciones eran $79,9 \%$ de biomasa de fuste y $20,3 \%$ de biomasa de ramas; en este estudio, si no se considera la fracción hojas las proporciones son $64,5 \%$ de biomasa de fuste y $35,5 \%$ de biomasa de ramas.

En el caso del roble, las fracciones se agrupan en una proporción de biomasa de fuste de $66,2 \%$ y de biomasa de copa de $33,8 \%$. Estos resultados son acordes con los obtenidos en otros estudios para especies del mismo género en España (Rapp et al., 1999; Gracia et al., 2002) y Portugal (Carvalho y Parresol, 2003), pero difieren de los obtenidos en estudios realizados en Centro Europa para Quercus petraea (André y Ponette, 2003) y en Norteamérica para Q. alba L., Q. coccinea Muenchh., Q. prinus Willd. y Q. rubra L. (Martin et al., 1998), en los que la biomasa de fuste representaba $73 \%$ a $84 \%$ de la biomasa aérea total.

En este estudio no se han desarrollado ecuaciones de estimación de biomasa de raíces debido al enorme esfuerzo de muestreo que supone la toma de datos. Ecuaciones de este tipo fueron desarrolladas para B. pubescens por Balboa-Murias et al. (2005) y para Q. robur por BalboaMurias et al. (2006). Pese a que el número de observaciones utilizado fue muy reducido (6 datos para abedul y 11 para roble), es la única información disponible por el momento.

\section{CONCLUSIONES}

Las ecuaciones desarrolladas en este estudio permiten estimar con suficiente precisión los pesos secos de las distintas fracciones consideradas y la biomasa aérea total. Éstas podrán ser empleadas con varios fines, como la cuantificación del potencial de las dos especies para el almacenamiento de carbono a medio y largo plazo en los montes de Galicia, o el posible aprovechamiento energético de los residuos (fundamentalmente los extraídos de la copa) que se generan en las operaciones silvícolas intermedias (aclareos, claras y podas) y en el aprovechamiento final.

El conjunto de datos utilizado en este estudio -significativamente superior al que existía hasta el momento- y las mejoras metodológicas en el caso de abedul podrían explicar los mejores resultados obtenidos en la fase de ajuste con respecto a estudios previos para estas especies en Galicia. Por lo tanto, se recomienda la utilización de las ecuaciones desarrolladas en este estudio en sustitución de las ecuaciones existentes.

\section{RECONOCIMIENTOS}

El presente estudio se enmarca dentro del proyecto "Modelos de evolución de bosques de frondosas autóctonas del noroeste peninsular" (AGL2007-66739-C02-01) financiado por el Gobierno de España a través del Ministerio de Ciencia e Innovación y cofinanciado por la Unión Europea mediante fondos FEDER.

\section{REFERENCIAS}

André, F. y Q. Ponette. 2003. Comparison of biomass and nutrient content between oak (Quercus petraea) and 
hornbeam (Carpinus betulus) trees in a coppice-with-standards in Chimay (Belgium). Annals of Forest Science 60:489-502.

Balboa-Murias, M.A., J.G. Álvarez González, A. Merino García y M. Barrio Anta. 2004. Revisión y discusión metodológica para la determinación de la biomasa forestal. Cuadernos de la Sociedad Española de Ciencias Forestales 18:35-39.

Balboa-Murias, M.A., A. Rojo, M.A Cela y G. Montero. 2005. Cuantificación del potencial de Betula alba L. para el almacenamiento de carbono a medio y largo plazo en Galicia. In: Actas del IV Congreso Forestal Español, Zaragoza (España), 26-30 septiembre.

Balboa-Murias, M.A., A. Rojo, J.G. Álvarez-González y A. Merino. 2006. Carbon and nutrient stocks in mature Quercus robur L. stands in NW Spain. Annals of Forest Science 63:557-565.

Belsley, D.A. 1991. Conditioning diagnostics, collinearity and weak data in regression. Wiley. Nueva York. 396 p.

Bond-Lamberty, B., C. Wang y S.T. Gower. 2002. Aboveground and belowground biomass and sapwood area allometric equations for six boreal tree species of northern Manitoba. Canadian Journal of Forestry Research 32:1441-1450.

Borders, B.E. 1989. Systems of equations in forest stand modelling. Forest Science 35:548-556.

Burger, H. 1945. Holz, Blattmenge und Zuwachs. VII: Die Lärche. Mitt Schw Anst forstl Forstl Versw 24:7-103.
Burger, H. 1953. Holz, Blattmenge und Zuwachs. XIII: Fichten im gleichaltrigen Hochwald. Mitt Schw Anst forstl Forstl Versw 29:38-130.

Carvalho, J.P. y B.R. Parresol. 2003. Additivity in tree components biomass of Pyrenean oak (Quercus pyrenaica Willd.). Forest Ecology and Management 179:269-273.

Cienciala, E., M. Cerný, J. Alptauer y Z. Exnerová. 2005. Biomass functions applicable to European beech. Journal of Forest Science 51(4):147-154.

Clark, A. 1982. Predicting biomass production in the South. In: Hotvedt, J.E. y B.D. Jackson, eds. Predicting growth and yield in the Mid-South. $31^{\text {th }}$ Annual For. Symp. Louisiana State University, Baton Rouge, L.A. p:119-139.

Cunia, T. 1986. Construction of tree biomass tables by linear regression techniques. In: Estimating tree biomass regressions and their error. Proceedings of the Workshop on tree biomass regression functions and their contribution to the error of forest inventory estimates. 26-30 Mayo. Siracusa, Nueva York. p:27-37.

Diéguez-Aranda, U., A. Rojo Alboreca, F. Castedo-Dorado, J.G. Álvarez González, M. Barrio-Anta, F. CrecenteCampo, J.M. González González, C. Pérez-Cruzado, R. Rodríguez Soalleiro, C.A. López-Sánchez, M.A. Balboa-Murias, J.J. Gorgoso Varela y F. Sánchez Rodríguez. 2009. Herramientas silvícolas para la gestión forestal sostenible en Galicia. Xunta de Galicia. Santiago de Compostela. $259 \mathrm{p}$. 
Gracia, C., S. Sabaté, J. Vayreda y J. Ibáñez. 2002. Aboveground Biomass Expansion Factors and biomass equations of forest in Catalonia. In: COST E21 BEF meeting, Besalú (España), 4-5 julio.

Huang, S. 2002. Validating and localizing growth and yield models: procedures, problems and prospects. In: Proceedings of IUFRO Workshop on Reality, models and parameter estimation: the forestry scenario. Sesimbra (Portugal), 2-5 junio.

Jian, R., C. Wang, B. Hawkins y T. Letchford. 1998. Relative growth rate and biomass allocation of paper birch (Betula papyrifera) populations under different soil moisture and nutrient regimes. Canadian Journal of Forestry Research 28:44-55.

Johansson, T. 2000. Biomass equations for determining functions of common and grey alder growing on abandoned farmland and some practical implicatons. Biomass and Bioenergy 18:147-159.

Jokela, E.J., C.A. Shannon y E.H. White. 1981. Biomass and nutrient equations for mature Betula papyrifera Marsh. Canadian Journal of Forestry Research 11:298-304.

Maliondo, S.M., M.K. Mahendrappa y G.D. van Raalte. 1990. Distribution of biomass and nutrients in some new brunswick forest stands: possible implications of whole-tree harvesting. Information Report M-X.170E/F. Hull, Quebec, Canadá.

Marklund, L.G. 1988. Biomassafunktioner för tall, gran och björk i Sverige. Sveriges lantbruksuniversitet, Institutionen för skogstaxering, Rapport 45:1-73.
Martin, J.G., B.D. Kloeppel, T.L. Schaefer, D.L. Kimbler y S.G. Mcnulty. 1998. Aboveground biomass and nitrogen allocation of ten deciduous southern Appalachian tree species. Canadina Journal of Forestry Research 28:1648-1659.

Mendoza Rivera, H., G.R. Bautista Mendoza, L.A. López y J.A. Vargas. 2002. Métodos de regresión, $1^{\text {a }}$. ed. Universidad Nacional de Colombia, Dirección Nacional de Innovación académica, Facultad de Ciencias, Departamento de Estadística, Bogotá, Col.

Merino, A., C. Rey, J. Brañas y R. Rodríguez-Soalleiro. 2003. Biomasa arbórea y acumulación de nutrientes en plantaciones de Pinus radiata D. Don en Galicia. Forest Systems 12(2):8598.

Montero, G., R. Ruiz-Peinado y M. Muñoz. 2005. Producción de biomasa y fijación de $\mathrm{CO}_{2}$ por los bosques españoles. Monografías INIA, Serie Forestal 13. Madrid. $270 \mathrm{p}$.

Myers, R.H. 1990. Classical and Modern Regression with Applications. $2^{a}$ ed. Duxbury Press, Belmont, CA. 488 p.

Návar, J.J., N. González, J.J. Graciano, V. Dale y B. Parresol. 2004. Additive biomass equations for pine species of forest plantations of Durango, Mexico. Madera y Bosques 10(2):1728.

Neter, J., M.H. Kutner, C.J. Nachtsheim y W. Wasserman. 1996. Applied linear statistical models. 4 ${ }^{\mathrm{a}}$ ed. McGrawHill. Nueva York. 739 p.

Pardé, J.D. 1980. Forest Biomass. Forestry Abstracts 41(8):343-362. 
Park, R.E. 1966. Estimation with Heteroscedastic Error Terms. Econometrica 34(4):888.

Parresol, B.R. 1999. Assessing tree and stand biomass: a review with examples and critical comparisons. Forest Science 45:573-593.

Parresol, B.R. 2001. Additivity of nonlinear biomass equations. Canadian Journal of Forestry Research 31:865-878.

Peña, D. 2002. Regresión y diseño de experimentos. Alianza Editorial, Madrid. 744 p.

Porté, A., P. Trichet, D. Bert y D. Loustau. 2002. Allometric relationships for branch and tree woody biomass of maritime Pine (Pinus pinaster Ait.). Forest Ecology and Management 158:71-83.

Rapp, M., I. Santa Regina, M. Rico y H.A. Gallego. 1999. Biomass, nutrient content, litterfall and nutrient return to the soil in Mediterranean oak forest. Forest Ecology and Management 119:39-49.

Ryan, T.P. 1997. Modern regression methods. John Wiley \& Sons, Nueva York. 515 p.

SAS Institute Inc. 2008. SAS/ETS ${ }^{\circledR}$ 9.2. User's Guide. SAS Institute Inc., Cary, NC. 2861 p.

SAS Institute Inc. 2009. SAS/STAT ${ }^{\circledR} 9.2$ User's Guide ( $2^{\text {nd }}$ ed.). SAS Institute Inc., Cary, NC. 7869 p.

Satoo, T. y H.A.I. Madgwick. 1982. Forest Biomass. Forestry Sciences. Kluwer Academic Publishers Group, Holanda. 160 p.
Schlaegel, B.E. 1982. Acer negundo biomass component regression analysis for the Mississipi Delta. Forest Science 28:355-358.

Schmitt, M.D.C. y D.F. Grigal. 1981. Generalized biomass equations for Betula papyrifera Marsh. Canadian Journal of Forestry Research 11:837-840.

Snowdon, P. 1985. Alternative sampling strategies and regression models for estimating forest biomass. Australian Forestry Research 15:353-366.

UNFCCC. 1997. The Kyoto Protocol. [online]. UNFCCC (United Nations Framework Convention on Climate Change). Disponible en http://unfccc. int/resource/docs/ convkp/kpeng. html. [2 de enero de 2012].

Wang, C. 2006. Biomass allometric equations for 10 co-occurring tree species in Chinese temperate forest. Forest Ecology and Management 222:9-16.

Waring, R.H. y S.W. Running. 1998. Forest ecosystems: analysis at multiple scales. $2^{a}$ ed. Academic Press. San Diego, CA. 370 p.

White, H. 1980. A heteroskedasticity-consistent covariance matrix estimator and a direct test for heteroskedasticity. Econometrica 48(4):817-838.

Zeide, B. 1987. Areas of biomass research. In: Wharton, E.H. y T. Cunia, eds. Estimating tree biomass regressions and their error. Proceedings of the Workshop on Tree biomass regression functions and their contribution to the error of forest inventory estimates. USDA For Serv Gen Tech Rep. p:193-196. 
Zellner, A. 1962. An efficient method of estimating seemingly unrelated regressions and test for aggregation bias. Journal of the American Statistical Association 57:348-368.
Zianis, D., P. Muukkonen, R. Mäkipää y M. Mencuccini. 2005. Biomass and stem volume equations for tree species in Europe. Silva Fennica, Monograps 4. $63 \mathrm{p}$. pubescens Ehrh.) y roble (Quercus robur L.) en el Noroeste de España. Madera y Bosques 19(1):71-91. 